\title{
Estimación de la edad dental en un grupo de niños venezolanos utilizando el método de Nolla
}

\section{Resumen}

La edad dental es un indicador de maduración corporal de importancia clínica en Odontología para la planificación del tratamiento de pacientes en crecimiento. El objetivo de esta investigación fue establecer la precisión de la determinación de la edad dental calculada con el método Nolla, en un grupo de niños y niñas del Área Metropolitana de Caracas. Materiales y Métodos: Se seleccionó una muestra estratificada por edad y género incluyendo 238 radiografías panorámicas de elevada calidad de personas sanas con edades entre 5 y 13 años. Se calculó la diferencia entre la edad cronológica y dental distribuyéndola entre géneros y grupos de edad. Los datos fueron analizados utilizando pruebas de correlación (Pearson), y diferencias entre medias $\mathrm{t}$-Student, ANOVA, fijando $\mathrm{p}<0,05$. El coeficiente k intraobservador fue de 0,68. Resultados: $\mathrm{La}$ correlación entre la edad dental cronológica y la edad dental fue de 0,92 años $(p=0,01)$. La formación dental se presentó retrasada en varones al compararla con la de las hembras, alcanzando significación estadística sólo para los caninos y primer premolar superior. Para el total, la diferencia entre la edad dental y cronológica fue estadísticamente significativa, registrándose una subestimación de la edad en $-0,88 \pm 0,94$ años $(\mathrm{p}=0,01)$. La subestimación fue mayor para las hembras $(-1,04 \pm 0,93$ años) que para los varones $(-0,72 \pm 0,94$ años). Conclusión: Se concluye que el Método de Nolla puede ser utilizado para la identificación de etapas de formación dental en la población de niños y niñas venezolanos, aunque carece de precisión para el cálculo de su edad dental.

Palabras clave: Determinación de la Edad pelos dientes, Niños, Radiografía Panorámica.

\section{Artigo Original}

\section{Estimativao da idade dentária em um grupo de crianças veneluelanas utilizando o método de nolla}

\section{Resumo}

A idade dentária é um indicador de maturação corporal de importância clínica em Odontologia para o planejamento do tratamento de pacientes em crescimento. $\mathrm{O}$ objetivo desta investigação é estabelecer a precisão na estimativa da idade dentária calculada com o método Nolla em

\footnotetext{
${ }^{1}$ Especialista en Odontopediatría, MSc. Profesor Asociado del Departamento de Odontología Pediátrica y Ortodoncia de la Facultad de Odontología. Universidad Central de Venezuela.

${ }^{2}$ Especialista en Bioestadística PhD. Profesor Titular de la Facultad de Medicina. Universidad Central de Venezuela.
} 
um grupo de meninos e meninas da área metropolitana de Caracas. Materiais e Métodos: Selecionou-se uma amostra estratificada por idade e gênero incluindo 238 radiografias panorâmicas de alta qualidade de pessoas saudáveis com idade entre 5 e 13 anos. Calculou-se a diferença entre a idade cronológica e dentária distribuindo-a de acordo com o gênero e grupo etário. Os dados foram analisados utilizando o coeficiente de correlação (Pearson) e as diferenças entre médias ( $t$-Student, ANOVA) fixando $p<0,05$. $O$ coeficiente $K$ intraobservador foi de 0,68. Resultados: A correlação entre a idade dentária cronológica e a idade dentária foi de 0,92 anos $(p=0,01)$. A formação dentária apresentou atraso nos meninos quando comparadoa com as meninas, sendoestatisticamente significante apenas paras canino e primeiro pré-molar superior. Para o total, a diferença entre a idade dentária e cronológica foi estatisticamente significante, registrando-se uma subestimativa da idade em $-0,88 \pm 0,94$ anos $(p=0,01)$. A subestimativa foi maior para as meninas $(-1,04 \pm 0,93$ anos) que para os meninos (-0,72 $\pm 0,94$ anos). Conclusão: Conclui-se que o Método de Nolla pode ser utilizado para a identificação de etapas da formação dentária na população de meninos e meninas venezuelanos, ainda que não tenha precisão para o cálculo da idade dentária.

Palavras chave: Determinação da Idade pelos Dentes, Criança, Radiografia Panorâmica

\section{Original article}

\section{Accuracy of dental age estimation in venezuelan children using the Nolla method}

\begin{abstract}
Dental age is a somatic maturation indicator with clinical importance for treatment planning in the growing patient. The purpose of this study is to determine the accuracy of the method proposed by Nolla for dental age assessment in a group of Venezuelan children from the Caracas Metropolitan area. Methods and materials: Good quality panoramic radiographs of $238 \mathrm{Ve}-$ nezuelan children aged 5-13 years were used to assess dental age using the method described by Nolla. The sample was stratified by age and gender. Mean differences between dental age and chronological age by gender and age groups were estimated (Perason's correlation, ANOVA, Student tests $\mathrm{p}=0.05)$. Intraobservar Kappa was 0.68. Results: Correlation between chronolo-
\end{abstract}

gical and dental age was $0.92(\mathrm{p}=0.01)$. Dental maturation was delayed in boys when compared to girls, with statistical significance for canines and maxillary first premolar. Difference between dental age and chronologic showed underestimation of $-0.88 \pm 0.94(p=0.01)$ which was statistically significant for the entire study group. Underestimation was larger for females $(-1.04 \pm 0.93$ years $)$ than for males $(-0.72 \pm 0.94$ years). Conclusion: The Nolla method may be accurately used for identifying dental formation stages by age group in Venezuelan children, but lacks accuracy for determining dental age, as underestimation was statistically significant for this study group.

Keywords: Age Estimation, Childrens, Panoramic radiographyc. 


\section{Introducción}

La edad fisiológica, biológica o de desarrollo, indica el progreso del individuo hacia el logro de la maduración completa. La estimación de la edad juega un papel importante en diversas áreas como medicina forense, endocrinología pediátrica, arqueología y odontología clínica. ${ }^{1-11}$ Ésta es estimada por medio de la evaluación de la maduración de uno o más sistemas de tejidos: sistema óseo, desarrollo de caracteres sexuales secundarios, masa corporal o sistema dental ${ }^{1}$. La edad dental puede ser determinada según la emergencia de los dientes o el grado de formación que éstos presenten, observados radiográficamente. ${ }^{5}$ De los métodos utilizados para la estimación de la edad biológica, los parámetros dentales han probado ser de gran utilidad en niños ya que la variabilidad en tasa de calcificación dental es baja, debido a que estos procesos están regidos principalmente por controles genéticos en lugar de factores ambientales, siendo los métodos más utilizados en la actualidad los que evalúan el desarrollo dental observado en radiografías panorámicas. ${ }^{11}$

Desde el punto de vista odontológico, la planificación del tratamiento tanto odontopediátrico como ortodóncico, puede ser influenciada por la determinación de la maduración dental. Las terapias preventivas, restauradoras, endodónticas y planificación de exodoncias en el paciente en crecimiento deben planificarse de acuerdo a la etapa de su dentición, considerando la maduración dental y los cambios esperados en el tiempo. Para el tratamiento ortodóncico debe incluirse el estudio del crecimiento y desarrollo del complejo dentofacial en particular y del cuerpo en general. ${ }^{12}$ La prevención e intercepción temprana de las deformidades dentofaciales depende de una correcta interpretación del crecimiento y desarrollo craneofacial en su conjunto, especialmente cuando la terapia propuesta es más eficaz en relación a la tasa de crecimiento o a la cantidad de crecimiento remanente, como ocurre con el uso de tracción extraoral, aparatos de ortopedia funcional, la decisión de extracciones, la retención y la planificación de cirugía ortognática. ${ }^{13}$

Considerando la edad dental como un indicador de la maduración corporal, cualquier variación en más de una desviación estándar, debe llamar la atención al odontopediatra ya que puede ser un signo temprano de alteraciones hormonales, metabólicas, nutricionales o sindrómicas, y se hace necesaria la interconsulta con el médico pediatra o endocrino pediatra. ${ }^{14} \mathrm{El}$ retardo de erupción debe alertar al clínico a realizar un examen más profundo ya que, después de haber descartado factores locales, puede haber un desequilibrio sistémico o de la fisiología del complejo craneofacial. ${ }^{15}$

El primer estudio longitudinal, realizado de manera sistemática sobre la calcificación y maduración de la dentición permanente mediante métodos radiográficos es el realizado por Carmen Nolla en $1960^{1}$. Esta autora destaca que la maduración dental, medida a través de la apreciación de la calcificación de los gérmenes en imágenes radiográficas tomadas a diferentes edades, permite la realización de un índice de determinación de edad dental y contribuye a la evaluación del organismo como un todo. Nolla estudió sistemáticamente la maduración de los gérmenes dentales, asignándole valores a cada etapa observada, construyendo tablas con las medias de maduración de cada diente por edad y género y tablas predictivas de la edad dental utilizando la sumatoria de las mismas. Éste es el primer estudio longitudinal y sistemático de cuantificación de la maduración de cada diente que, mediante la aplicación de cálculos realizados sobre una 
muestra, permitió la elaboración de estimaciones de la edad basada en evidencia. ${ }^{16}$

Nolla destaca que los estudios previos habían sido realizados por otros investigadores utilizando cortes histológicos, observación y radiografías de material de autopsia. Cita a Todd ${ }^{17}$, quien señala que un niño fallecido es un infante con un defecto en el que ha ocurrido la interrupción del crecimiento y desarrollo en algún momento antes de la muerte, a menos que ésta haya sido producto de una enfermedad aguda o de un accidente, hecho que debe tomarse en consideración al evaluar estos cráneos. De allí que para investigar individuos sanos, debe analizarse a los vivos. Por ello, la evaluación radiográfica toma especial importancia.

Nolla ${ }^{1}$ propuso los diferentes estadios del desarrollo de dientes permanentes y su relación con la maduración somática. La muestra de trabajo estuvo conformada por 25 varones y 25 hembras, a quienes se les realizaron radiografías anuales completando un total de 1746 para los varones y 1656 radiografías para las niñas. La investigadora examinó radiografías extraorales laterales derecha e izquierda, intraorales oclusales superior e inferior e intraorales periapicales de todos los molares. Las radiografías fueron obtenidas de los archivos de los Laboratorios de Desarrollo de la Infancia de la Universidad de Michigan. Se elaboró un sistema de 11 estadios de maduración para cada diente, entre 0 indicando ausencia de datos de calcificación del germen dental hasta 10, equivalente a cierre apical completo. Nolla observó que no había diferencia significativa de los estadios de maduración entre los lados derecho e izquierdo, por lo que los valores de un lado son representativos de los valores del lado contrario.

Nolla ${ }^{1}$ construyó gráficos de maduración representativos de los valores promedios de ma- duración para cada diente y determinó que las hembras demostraron iniciar y culminar el desarrollo dental más tempranamente que los varones, proponiendo tablas por género con la media de formación dental para cada diente en función de la edad cronológica. Para determinar la edad dental, debe sumarse el valor asignado a cada diente, obteniendo una puntuación directa de suma total de los dientes mandibulares y maxilares, incluyendo o no los terceros molares. Esos valores fueron registrados en tablas recopilatorias.

El sistema desarrollado por Nolla ha sido probado en algunas poblaciones, con resultados variables, ${ }^{7,18-21}$ observándose que, en la mayoría de los casos, a pesar de haber elevada correlación entre la edad dental y cronológica, la diferencia entre ellas es estadísticamente significativa. Sin embargo, la esquematización y asignación de valores a cada etapa de la dentición es de utilidad clínica para el estudio de la calcificación y maduración dental, en especial para comparar la etapa de formación de cada diente a una edad específica.

La presente investigación tiene como objetivo determinar la precisión del método de determinación de maduración dental de Nolla en un grupo de niños y niñas del Área Metropolitana de Caracas. Esto permitirá conocer su comportamiento en una muestra venezolana, determinar su aplicabilidad clínica y realizar recomendaciones en cuanto a su uso.

\section{Material y métodos}

Esta investigación es de tipo documental, transversal y correlacional y cuenta con el aval del Comité de Bioética de la Facultad de Odontología de la Universidad Central de Venezuela \#0112-2010. La población la constituyen el total 
de registros radiográficos de pacientes pediátricos diagnosticados y atendidos en una clínica odontológica privada. La muestra incluyó pacientes con edades de 5 a 13 años ambos inclusive, seleccionada de manera estratificada, teniendo especial cuidado de incluir al menos 15 radiografías correspondientes a varones y 15 a hembras, representativas de cada edad cronológica, en los que la edad se expresa en años cumplidos al momento de la toma radiográfica. Los criterios de inclusión incluyeron: Radiografías panorámicas de buena calidad, tomadas a niños y niñas aparentemente sanos, en las que se observaron todos los gérmenes dentales claramente. Estas radiografías fueron obtenidas previamente como parte del diagnóstico integral de los pacientes ${ }^{22,23}$, no se realizaron tomas específicas para la presente investigación. En todos los casos se visualizaron claramente ambos cóndilos, el borde inferior de ambas órbitas y el borde antero-inferior de la mandíbula y los incisivos no presentaban distorsión, según criterios establecidos para la observación de radiografías panorámicas. ${ }^{24}$

Mientras que los criterios de exclusión comprendieron: Radiografías de poca calidad, con distorsión o en las que no se observaran claramente los gérmenes dentales. Igualmente fueron excluidos los casos con historia de nacimiento prematuro, síndromes, enfermedades sistémicas o nutricionales, alteraciones de número dental, pérdidas prematuras de dientes primarios, tratamiento ortodóncico y antecedentes de traumatismos dentofaciales.

La información concerniente a edad de nacimiento y de toma de radiografías, así como género y estadio de formación dental se registró en fichas diseñadas para tal fin. Las radiografías se observaron en negatoscopio, con luz apropiada. Se tomaron fotografías digitalizadas con cámara
Nikkon 10 Megapixeles para analizar, utilizando programa Adobe ${ }^{\circledR}$ Photoshop ${ }^{\circledR}$ CS4 Versión 11.0 (C1990-2008 Adobe Systems Incorporated, Estados Unidos. Cada radiografía fue analizada por el mismo observador, con un máximo de 10 radiografías por sesión.

La edad cronológica se calculó en forma decimal restando la fecha de nacimiento de la fecha de la toma radiográfica. La edad legal corresponde a los años cumplidos (en enteros) al momento de la toma radiográfica. La determinación de la edad dental se hizo siguiendo el método descrito por Nolla ${ }^{1}$. Se determinó la etapa de formación radicular de los 7 dientes mandibulares y de los 7 dientes maxilares: incisivo central (I1), incisivo lateral (I2), Canino (C), primer premolar (PM1), segundo premolar (PM2), primer molar permanente (M1) y segundo molar permanente, primer molar permanente (M2), asignándoles el valor ponderado.

Para determinar el grado de desarrollo de cada diente se comparó su imagen con la figura comparativa que estuviera lo más próxima posible. Los valores asignados fueron $0=$ ausencia de cripta, $1=$ presencia de cripta, $=2$ calcificación inicial, $3=1 / 3$ corona completa, $4=2 / 3$ corona completa, $5=$ corona casi completa, $6=$ corona completa, $7=1 / 3$ de raíz, $8=2 / 3$ de raíz, $9=$ raíz casi complete, ápice abierto y $10=$ ápice radicular completo.

Si el dato radiográfico se encontraba entre dos posibles grados, se le asignó el menor de los dos agregándole 0,5. Cuando la imagen radiográfica fue ligeramente más grande se agregó 0,2 y si se aproximó mucho al valor siguiente, se le sumó 0,7 .

Se añadieron los valores ponderados y el resultado se comparó con la tabla de estimación de 
edad dental, obteniendo la misma. Posteriormente, se comparó la edad cronológica con la edad dental estimada.

Todas las observaciones fueron realizadas por un mismo investigador (ACM). Para la determinación de la concordancia intraobservador de las etapas de formación se realizó el cálculo del coeficiente Kappa. ${ }^{25}$ Para ello, se escogieron 28 pacientes seleccionados de forma estratificada por edad y género. Las variables de formación dental fueron registradas dos veces por el mismo observador, en días diferentes con un máximo de 10 radiografías por sesión. Se obtuvo un Kappa intraobservador de 0,68.

Los datos fueron analizados utilizando métodos descriptivos e inductivos. Las diferencias entre edad cronológica y edad dental estimada, así como las diferencias entre géneros fueron analizadas con la prueba t de Student. La asociación entre edad cronológica y dental fue analizada a partir de la correlación de Pearson. Los métodos de Kruskall-Wallis y ANOVA fueron utilizados para determinar las diferencias entre medias por grupo de edad. Se probó la normalidad y homo- geneidad de los datos. Se obtuvo consistencia para los niveles de significancia entre las pruebas paramétricas y no paramétricas, mostrando los resultados obtenidos mediante aplicación de pruebas paramétricas. Se fijó un intervalo de confianza de $95 \%$ para determinar significación estadística.

La información recogida se transcribió y tabuló en programa Microsoft® Office Excel 2007 (C) 2008 Microsoft Corporation, Estados Unidos para luego ser procesados con software estadístico PASW® Statistics 18 (SSPS 18), (C) 2009 SPSS Inc., EEUU.

\section{Resultados}

La muestra total estuvo constituida por 238 radiografías panorámicas, 117 varones y 121 hembras. Para cada género, el porcentaje de individuos por grupo de edad fue muy similar, con excepción de los grupos de 11, 12 y 13 años, en los que, al aplicar los criterios de exclusión, disminuyó su número (Tabla I). La edad cronológica promedio fue de 8,86 $\pm 2,34$ años.

Tabla I. Distribución de la muestra según edad y género.

\begin{tabular}{|c|c|c|c|c|c|c|}
\hline Edad & Femenino & $\%$ & Masculino & $\%$ & Total & $\%$ \\
\hline 5,00 a 5,99 & 16 & 13,2 & 15 & 12,8 & 31 & 13,0 \\
\hline 6,00 a 6,99 & 16 & 13,2 & 16 & 13,7 & 32 & 13,5 \\
\hline 7,00 a 7,99 & 15 & 12,4 & 16 & 13,7 & 31 & 13,0 \\
\hline 8,00 a 8,99 & 16 & 13,2 & 15 & 12,8 & 31 & 13,0 \\
\hline 9,00 a 9,99 & 17 & 14,0 & 16 & 13,7 & 33 & 14,0 \\
\hline 10,00 a 10,99 & 17 & 14,0 & 14 & 12,0 & 31 & 13,0 \\
\hline 11,00 a 11,99 & 12 & 10,0 & 14 & 12,0 & 26 & 10,9 \\
\hline 12,00 a 12,99 & 8 & 6,7 & 4 & 3,4 & 12 & 5,0 \\
\hline $13,0013,99$ & 4 & 3,3 & 7 & 5,9 & 11 & 4,6 \\
\hline Total & 121 & 50,8 & 117 & 49,2 & 238 & 100 \\
\hline
\end{tabular}


Tabla II. Medias de formación radicular (etapas de Nolla) para la muestra.

\begin{tabular}{|c|c|c|c|c|c|c|c|c|c|c|c|c|c|c|c|}
\hline $\begin{array}{l}\text { EDAD } \\
\text { LEGAL }\end{array}$ & GÉNE & 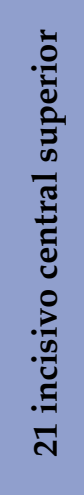 & 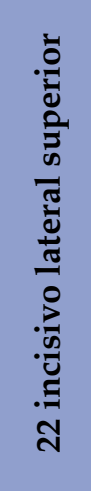 & 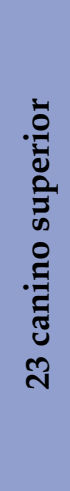 & 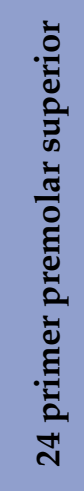 & 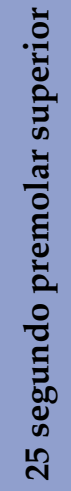 & 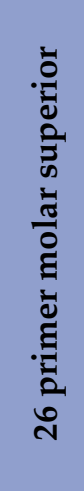 & 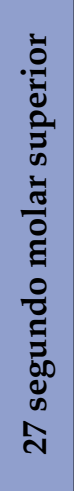 & 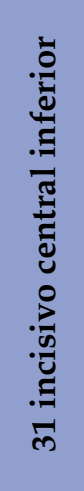 & 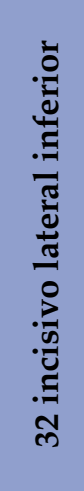 & 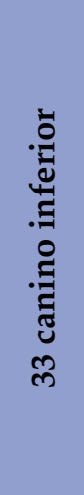 & 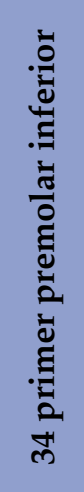 & 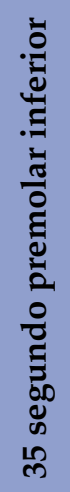 & 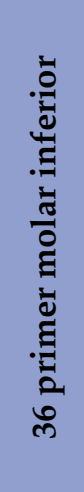 & 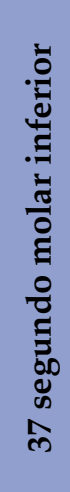 \\
\hline \multirow{2}{*}{$\begin{array}{c}5,00 \mathrm{a} \\
5,99\end{array}$} & & 6,9 & 6,4 & $6,($ & 5,4 & 4 & 7,1 & 3,8 & 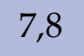 & 7, & & 3 & 3 & 4 & 3,7 \\
\hline & Masculino & 6,7 & 6,1 & 5,7 & 4,8 & 3,8 & 7,3 & 3,4 & 7,5 & 7,0 & $5 \mathrm{c}$ & 5,1 & 4,3 & 7,6 & 3,5 \\
\hline \multirow{2}{*}{$\begin{array}{c}6,00 \mathrm{a} \\
6,99\end{array}$} & $\Gamma$ & 7,7 & 7,1 & 6,5 & 6,0 & 5,1 & 8,4 & 5,0 & 8,7 & 8,0 & 6,7 & 6,1 & 5,3 & 8,5 & 4,6 \\
\hline & Masculino & 7,0 & 6,4 & 5,8 & 5,5 & 4,3 & 7,8 & 4,3 & 8,1 & 7,4 & 6.0 & 5,6 & 5,0 & 7,6 & 4,1 \\
\hline \multirow{2}{*}{$\begin{array}{c}7,00 \mathrm{a} \\
7,99\end{array}$} & $F$ & 8,2 & 7,7 & 7,1 & 6,2 & 5,8 & 8,6 & 5,2 & 9,2 & 8,7 & 7,2 & 6,3 & 5,6 & 8,8 & 5,1 \\
\hline & Masculino & 8,1 & 7,4 & 6,5 & 5,8 & 5,3 & 8,8 & 4,2 & 9,1 & 8,5 & 6,6 & 6,1 & 5,4 & 8,8 & 4,6 \\
\hline \multirow{2}{*}{$\begin{array}{c}8,00 \mathrm{a} \\
8,99\end{array}$} & $\mathrm{~F}$ & 9,1 & 8,6 & 7,7 & 7,0 & 6,5 & 9,3 & 5,6 & 9,8 & 9,0 & 7,8 & 7,1 & 6,5 & 9,3 & 5,9 \\
\hline & Masc & 8,9 & 8,5 & 7,3 & 6,8 & 6,4 & 9,1 & 5,8 & 9,2 & 9,3 & 7,5 & 6,9 & 6,3 & 9,3 & 5,8 \\
\hline \multirow{2}{*}{$\begin{array}{c}9,00 \mathrm{a} \\
9,99\end{array}$} & $\mathrm{Fe}$ & 9,6 & 9,1 & 8,2 & 7,8 & 7,3 & 9,6 & 6,3 & 9,9 & 9,7 & 8,5 & 8,0 & 7,4 & 9,7 & 6,5 \\
\hline & Masculino & 9,7 & 9,4 & 8,1 & 7,6 & 7,0 & 9,6 & 6,5 & 10,0 & 9,8 & 8,3 & 7,7 & 7,1 & 9,6 & 6,6 \\
\hline \multirow{2}{*}{$\begin{array}{c}10,00 \mathrm{a} \\
10,99\end{array}$} & Femenino & 10,0 & 10,0 & 9,0 & 8,5 & 8,1 & 9,9 & 7,2 & 10,0 & 10,0 & 9,0 & 8,9 & 8,4 & 9,9 & 7,6 \\
\hline & Masculino & 9,9 & 9,5 & 8,4 & 8,0 & 7,6 & 9,8 & 6,6 & 10,0 & 10,0 & 8,5 & 8,3 & 7,7 & 9,9 & 7,1 \\
\hline \multirow{2}{*}{$\begin{array}{c}11,00 \mathrm{a} \\
11,99\end{array}$} & Femenino & 10,0 & 9,8 & 9 & 9,3 & 8,7 & 10,0 & 7,9 & 10,0 & 10,0 & & 9,3 & 8,7 & 10,0 & 8,0 \\
\hline & Masculino & 9,9 & 9,9 & 8,8 & 8,6 & 0,1 & (נ) & 7,4 & 10,0 & 10,0 & 0,0 & 8,9 & 8,3 & 10,0 & , \\
\hline \multirow{2}{*}{$\begin{array}{c}12,00 \mathrm{a} \\
12,99\end{array}$} & Femer & 10,0 & 9,9 & 9, & 9,3 & 0,0 & 10,0 & 8,2 & 10,0 & 10,0 & , & 9,4 & 8,9 & 10,0 & 8,4 \\
\hline & Masculino & 10,0 & 10,0 & Y,3 & 9,5 & 9,1 & 10,0 & 8,0 & 10,0 & 10,0 & (3,0 & 9,5 & 9,1 & 10,0 & 8,8 \\
\hline \multirow{2}{*}{$\begin{array}{l}13,00 \\
13,99\end{array}$} & Femenino & 10,0 & 10,0 & Y,8 & 10,0 & 9,0 & 10,0 & 9,1 & 10,0 & 10,0 & 10,0 & 10,0 & 9,5 & 10,0 & 9,0 \\
\hline & Masculino & 10,0 & 10,0 & 9,6 & 9,4 & 9,4 & 10,0 & 9,3 & 10,0 & 10,0 & 9,6 & 9,7 & 9,4 & 10,0 & 9,3 \\
\hline
\end{tabular}

Las etapas de formación dental de Nolla fueron estudiadas, distribuyéndolas por edad legal y género. Los pacientes masculinos presentaron una formación radicular ligeramente menor para la mayoría de los dientes según la edad, esa diferencia es menor a media etapa en la mayor parte de los casos (Tabla II). Las diferencias en- tre las medias de formación dental del total de la presente muestra y las reportadas por Nolla no alcanzaron significación estadística. Por otro lado, las diferencias por género fueron estadísticamente significativas sólo para el canino inferior, el canino superior y el primer premolar superior (Tabla II). La sumatoria de las etapas 


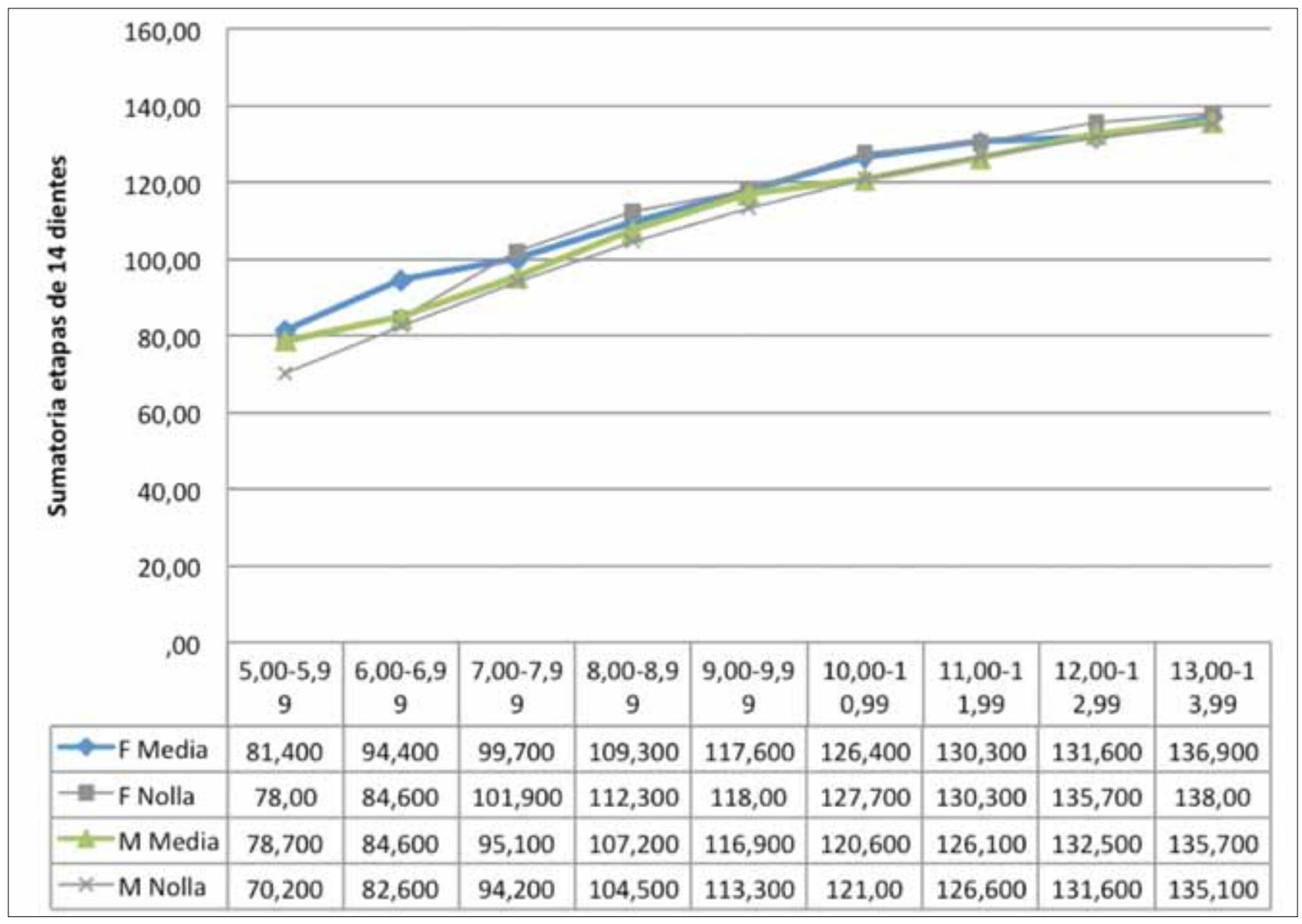

Fig. 1. Media de la sumatoria de etapas de 14 dientes (7 maxilares y 7 mandibulares) por edad, comparada con las medias propuestas por Nolla ${ }^{1}$ (F Media= Medias para el sexo Femenino en la presenta muestra, F Nolla= medias de Nolla para sexo femenino, M Media= Medias para el sexo Masculino en la presenta muestra, M Nolla= medias de Nolla para sexo masculino).

de formación de los 14 dientes evaluados se distribuyó por edad y género, y se compararon con las obtenidas por Nolla. Para las hembras a edades tempranas, la media obtenida fue mayor que la propuesta por Nolla, sin embargo, a partir de los 7 años de edad, la sumatoria fue menor, aunque sin significación estadística. Para los varones, la media de sumatoria obtenida fue similar a la planteada por Nolla, siendo un poco mayor hasta los 9 años de edad y menor a partir de los 10 años de edad (Figura 1).

Ahora bien, se obtuvo una alta asociación entre la edad dental según Nolla y edad cronológica (rho $=0,92, \mathrm{p}=0,01$ ).
Las edades cronológicas y edades dentales según Nolla, así como la media y la desviación estándar de sus diferencias se describen en la Tabla III. En todos los grupos etarios, el método de Nolla subestimó la edad, por lo que la edad dental fue siempre menor que la edad cronológica actual del paciente. La diferencia fue mayor al aumentar la edad y su rango fluctúo entre 6 meses y un año y tres meses. La media de la diferencia entre la edad dental y la edad cronológica fue analizada según género (Figura 2). La subestimación de la edad fue mayor en el género femenino, siendo cercana a -1 año entre los 7 y 11 años de edad y alcanzando un promedio de $-1,7$ años a los 12 años (-1,04 \pm 0,93 años). La 
Tabla III. Edad cronológica y edad dental calculada utilizando el método de Nolla (diferencia y desviación estándar DS, distribuidas según edad).

\begin{tabular}{|c|c|c|c|c|}
\hline $\begin{array}{l}\text { Edad Legal } \\
\text { (años) }\end{array}$ & $\begin{array}{c}\text { Media Edad } \\
\text { Cronológica } \\
\text { (años) }\end{array}$ & $\begin{array}{c}\text { Media Edad } \\
\text { Dental Nolla } \\
\text { (años) }\end{array}$ & $\begin{array}{l}\text { Diferencia } \\
\text { (años) }\end{array}$ & $\begin{array}{c}\text { DS } \\
\text { (años) }\end{array}$ \\
\hline 5,00 a 5,99 & 5,54 & 4,97 & $-0,573$ & 0,105 \\
\hline 6,00 a 6,99 & 6,50 & 5,78 & $-0,720$ & 0,110 \\
\hline 7,00 a 7,99 & 7,37 & 6,42 & $-0,954^{* *}$ & 0,127 \\
\hline 8,00 a 8,99 & 8,50 & 7,55 & $-0,947^{* *}$ & 0,153 \\
\hline 9,00 a 9,99 & 9,46 & 8,58 & $-0,889^{*}$ & 0,158 \\
\hline 10,00 a 10,99 & 10,47 & 9,48 & $-0,989^{\star *}$ & 0,127 \\
\hline 11,00 a 11,99 & 11,54 & 10,46 & $-1,082^{* *}$ & 0,231 \\
\hline 12,00 a 12,99 & 12,62 & 11,33 & $-1,284$ & 0,428 \\
\hline $13,0013,99$ & 13,52 & 12,91 & $-0,615$ & 0,639 \\
\hline Total & & & $-0,883^{* *}$ & 0,947 \\
\hline \multicolumn{5}{|c|}{ Prueba ANOVA post hoc Tuckey ${ }^{* *} \mathrm{p} \leq 0,01{ }^{*} \mathrm{p} \leq 0,05$} \\
\hline
\end{tabular}

diferencia para el género masculino fue cercana a $-0,7$ años entre los 7 y los 11 años. Se observa que a medida que se avanza en edad, mayor es la subestimación (-0,72 \pm 0,94 años) (Figura 2). La diferencia entre géneros alcanzó significación estadística $(\mathrm{p}<0,05)$.

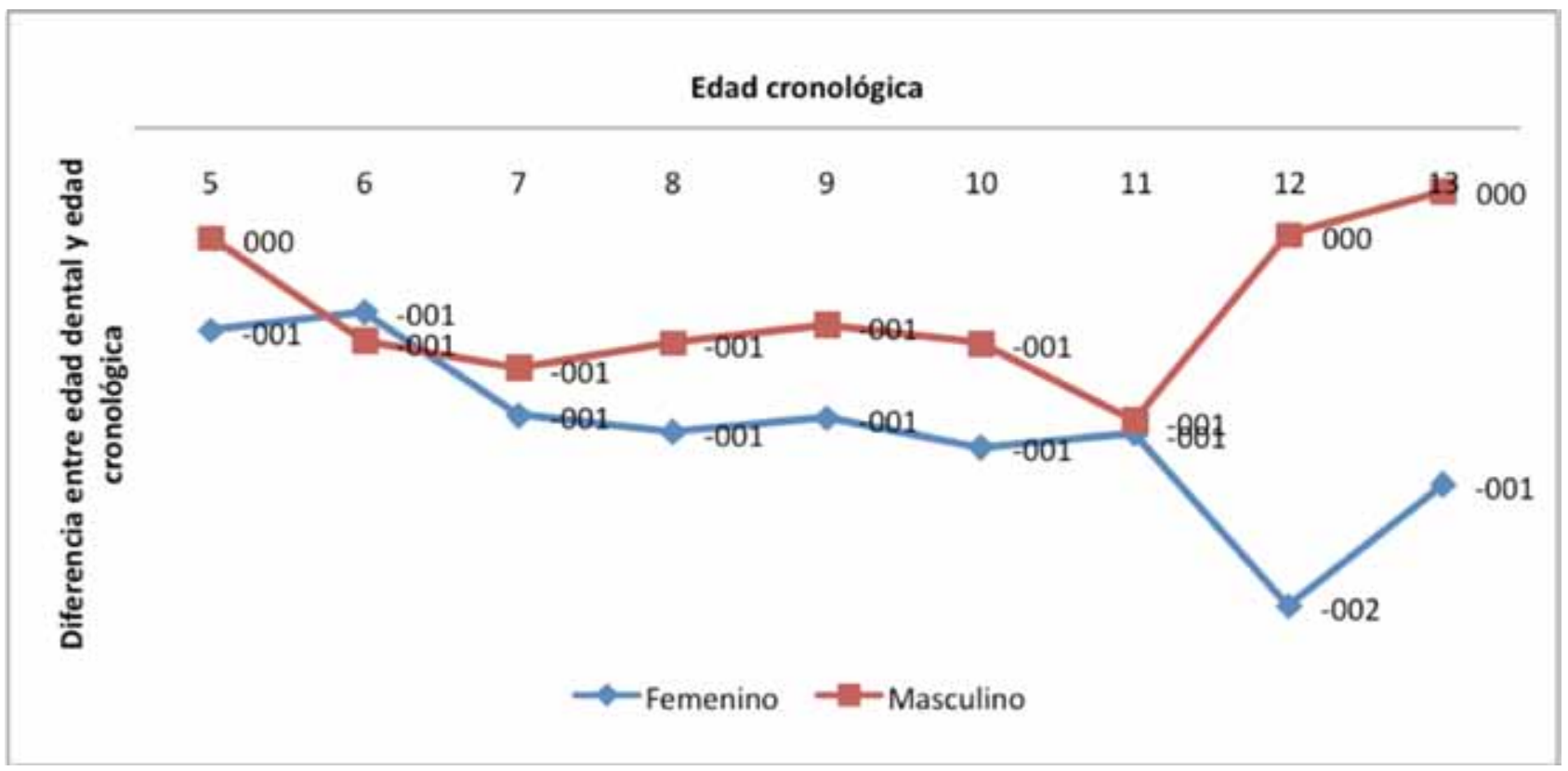

Fig. 2. Media de las diferencias entre edad cronológica y edad dental calculada con el método de Nolla, distribuido por edad y género. 


\section{Discusión}

La edad dental es un indicador de maduración corporal de importancia en varios ámbitos, tanto legal como médico, donde la planificación del tratamiento odontológico del paciente en crecimiento reviste especial interés. Se han descrito diversos métodos de estimación de la maduración dental, los cuales han presentado resultados variables en las distintas poblaciones donde han sido aplicados.

En este estudio se seleccionaron radiografías de alta calidad de pacientes en crecimiento para aplicar el método de estimación de maduración dental. El rango de edad fue de 5 a 13 años inclusive, abarcando las edades en las cuales éstas se realizan rutinariamente y en las que mayor cantidad de cambios se observan en el desarrollo dental. La distribución observada por edad y género fue similar, observándose una menor cantidad de pacientes de 12 y 13 años, producto de la exclusión de casos con antecedentes de tratamiento ortodóncico o exodoncias. Ahora bien, es de destacar que en esas edades la cantidad de etapas que se observan en los dientes en formación desciende. Esto se debe a que cerca de los 10 años de edad muchos dientes han alcanzado el cierre apical, disminuyendo el número de variables a evaluar en edades posteriores, según plantean Maber y Cols. ${ }^{7}$

Los criterios de exclusión en la selección de la muestra fueron muy rigurosos, tomando en consideración los factores conocidos que afectan el desarrollo de la dentición, como nacimiento prematuro, ${ }^{26}$ factores nutricionales ${ }^{27}$ e historia de tratamiento endodóncico o pérdidas prematuras de dientes primarios. ${ }^{28,29}$

El uso de este método no ha sido ampliamente reportado en la literatura a pesar de que las eta- pas de formación que se plantean son fácilmente identificables y reproducibles. La utilización de identificación numérica da cada etapa facilita los cálculos estadísticos, además de permitir la asignación de decimales cuando existe duda entre dos etapas.

Nolla ${ }^{1}$ plantea diferencias en el cálculo de la edad dental entre géneros. En la presente muestra estas diferencias fueron observadas para todas las etapas, en cada diente, siendo estadísticamente significativas para el canino superior y para el canino y el primer premolar inferior. De esta manera se constata que la formación radicular es diferente entre géneros para una misma edad legal.

Para cada grupo de edad, se comparó la formación radicular media observada en la presente muestra con la planteada por Nolla. Hubo diferencias pequeñas, siendo en algunos casos muy próxima, siendo mayor la etapa observada en la muestra que la reportada por Nolla. De allí que se plantea que el uso de la tabla de medias de maduración dental por edad planteada por Nolla, puede ser utilizada como referencia para esta población.

Por otro lado, las sumatorias por edad, según género, fueron muy similares, siendo ligeramente mayor para las hembras. Al comparar la edad dental y la edad cronológica se observó subestimación para todas las edades, siendo su promedio $-0,88 \pm 0,95$ años. Esta diferencia fue estadísticamente significativa $(p=0,01)$. Sin embargo, al realizar la correlación entre edad dental y cronológica su magnitud fue elevada (rho=0,921; $\mathrm{p}=0,01)$. En el ámbito clínico, el uso de las tablas de maduración propuestas por No$1 a^{1}$ puede tener gran utilidad ya que permite determinar si un diente en particular presenta una etapa de desarrollo acorde con su edad cro- 
nológica. En la muestra estudiada se observó que las etapas de formación fueron similares a las reportadas por Nolla, planteándose que en esta población de niños venezolanos es posible utilizar las tablas de maduración de Nolla como referencia de maduración dental.

De forma similar a los hallazgos realizados en los niños venezolanos, Maber y Cols. ${ }^{7}$, en un grupo de 946 niños Caucásicos e Indianos en el Reino Unido, reportaron una subestimación estadísticamente significativa entre la edad cronológica y la edad dental de $-1,02 \pm 0,85$ años. Similarmente a la presente investigación, la subestimación hallada por Maber y Col. ${ }^{7}$ es más amplia en las hembras $(-1,18 \pm 0,96$ años) que en los varones $(-0,87 \pm 0,87$ años $)$.

Otro estudio con resultados similares a la presente investigación es el realizado por Kurita y Cols. ${ }^{20}$ en un grupo de 360 niños en Brasil, quienes reportaron que la correlación entre edad dental y cronológica fue de 0,87 para las hembras y 0,84 para los varones. Estos autores también encontraron que el método de Nolla sub- estima la edad, siendo esta subestimación más amplia para las hembras (-13,82 meses) que para los varones (-10,6 meses).

Hay diferencias con los resultados de otros estudios con muestras de menor tamaño, por ejemplo, con lo observado por Rai y Anand ${ }^{19}$ para 75 niños Indianos, en los que este método sobresti-

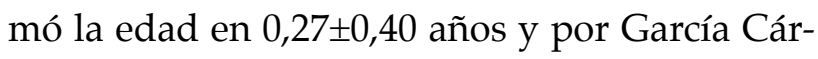
denas y Cols. ${ }^{18}$ en un grupo de 100 niños mejicanos con edades de 5 a 12 años, quienes hallaron una correlación menor a la reportada en el presente estudio $(0,55-0,80)$.

\section{Conclusión}

Por lo anteriormente expuesto, puede concluirse que el método de Nolla puede ser utilizado para la identificación de etapas de formación dental y su comparación con las tablas establecidas. Sin embargo, para la muestra investigada el mismo carece de precisión para el cálculo de la edad dental, dado que se produjo una subestimación significativamente importante.

\section{Referencias}

1. Nolla C. Development of the permanent teeth. J Dent Child. 1960; 27: 254-63.

2. Espina de Fereira A, Fereira J, Céspedes M, Barrios F, Ortega A, Maldonado Y. Empleo de la edad dental y la edad ósea para el cálculo de la edad cronológica con fines forenses en niños escolares con valores de talla y peso no comparables con su edad y sexo, en Maracaibo, Estado Zulia. Acta Odont Venez. 2007; 45 (1): 14-21.

3. Tineo F, Espina de Fereira A, Barrios F, Ortega A, Fereira J. Estimación de la edad cronológica con fines forenses, empleando la edad dental y la edad ósea en niños escolares en Maracaibo, Estado Zulia. Estudio preliminar. Acta Odont Venez. 2006; 44(2).

4. Cruz-Landeira A, Linares-Argote J, Martínez-Rodríguez M, Rodríguez-Calvo M, Otero X, Concheiro L. Dental age estimation in Spanish and Venezuelan children. Comparison of Demirjian and Chaillet's scores. Int J Legal Med. 2009; 124(2): 105-12. 5. Willems G. A review of the most commonly used dental age estimation techniques. J For Odonto Stomatol. $2001 ; 19: 9-17$.

6. Chaillet N, Nyström M, Demirjian A. Comparison of dental maturity in children of different ethnic origins: international maturity scores for clinicians. J Forensic Sci. 2005; 50(5): 1164-74.

7. Maber M, Liversidge H, Hector M. Accuracy of age estimation of radiographic methods using developing teeth. Forensic Sci Intl. 2006; S68-S70. 
8. Pérez Flores M, Feijoo García G, Llanes Gómez C, Maroto Edo M. Determinación de la edad dentaria. Implicaciones clínicas y legales. Rev Soc Chil Odontopediatría. 2007; 23(2): 5-14.

9. Čuković Bagić I, Server N, Brkić H, Kern J. Dental age estimation in children using orthopantograms. Acta Stomatol Croat. 2008; 421: 11-8.

10. El-Bakary A, Hammad S, Mohammed F. Dental age estimation in Egyptian children, comparison between two methods. J Forensic \& Legal Med. 2010;: doi:10.1016/j.flm.2010.05.08.

11. Maia M, Martins M, Germano F, Neto J, da Silva C. Demirjian's method for estimating the dental age of northeastern Brazilian children. Forensic Sci Intl. 2010;(20): 177.e1-177.e4.

12. Green L. The interrelationships among height, weight and cronological, dental and skeletal ages. Angle Orthod. 1961; 31(3): 189-93.

13. Flores-Mir C, Orth C, Nebbe B, Major P. Use of skeletal maturation based on hand-wrist radiographic analysis as a predictor of facial growth: a systematic review. Angle Orthod. 2004; 74: 118-24.

14. Gibson W, Conchie J. Observation of children`s teeth as a diagnostic aid: a review. Part I. Dentition in the assessment of development. Canad Med Ann J. 1964; 90: 70-5.

15. Suri L, Gagari E, Vastardis H. Delayed tooth eruption: pathogenesis, and treatment. A literature review. Am J Orthod Dentofacial Orthop. 2004; 126: 432-45.

16. Garamendi González P. Repositorio Institucional de la Universidad de Granada. [Online]. Granada; 2007 [cited 2010 Agosto 20. Available from: http:/ / hdl.handle.net/10481/1650.

17. Todd T. The orthodontic value of research and observations in development of the face. Angle Orthod. 1931; 1: 67-9.

18. García Cárdenas E, Hernández Morales S, Valencia Hitte R, Jiménez M. Correlación entre la edad cronológica y la edad dental en niños mexicanos de 5 a 12 años atendidos en el posgrado de odontología pediátrica en la Universidad Tecnológica de México. Rev AMOP. 2002; 14: 4-6.

19. Rai B, Anand S. Tooth development: an accuracy of age estimation of radiographic methods. World J Med Scien. 2006; 1(2): $130-2$.

20. Kurita L, Menezes A, Casanova M, Haiter-Neto F. Dental maturity as an indicator of chronological age: radiographic assessment of dental age in a Brazilian population. J Appl Oral Sci. 2007; 15(2): 99-104.

21. El-Yazed A, Zeid A, Tawfik W. Dental maturation assessment by Nolla's Technique on a group of Egyptian children. Aust J Basic \& Appl Sci. 2008; 2(4): 1418-24.

22. American Dental Association. Panoramic imaging. J Am Dent Assoc. 2002;(133): 1697-8.

23. American Association of Pediatric Dentistry. Guideline on prescribing dental radiographs for infants, children, adolescents, and persons with special needs. Pediatr Dent. 2010/11; Refernence Manual 32: 272-4.

24. White S, Heslop E, Hollander L, Mosier K, Ruprecht A, Shrout M. parameters of radiologic care: an official report of the American Academy of Oral and Maxillofacial Radiology. Oral Surg Oral Med Oral Pathol Oral Radiol Endod. 2001 ; 91: 498-511. 25. Cerda J, Villarroel L. Evaluación de la concordancia inter-observador en investigación pediátrica: Coeficiente de Kappa. Rev Chil Pediatr. 2008; 1: 54-8.

26. Seow W. Effects of preterm birth on oral growth and development. Austr Dent J. 1997; 42(2): 85-91.

27. Flores-Mir C, Mauricio F, Orellana M, Major P. Association between growth stunting with dental development and skeletal maturation stage. Angle Orthod. 2005; 75: 935-40.

28. Fanning E. Effect of extraction of deciduous molars on the formation and eruption of their successors. Angle orthod. 1962; 321: 44-53.

29. Haralabakis N, Yiagtzis S, Toutountzakis N. Premature or deleyed exfoliation of deciduous teeth and root resorption and formation. Angle Orthod. 1994; 64(2): 151-7.

Recibido: 09-10-2013

Aceptado: 10-11-2013

Correspondencia: Piso 6, Comisión de Estudios de Postgrado, Facultad de Odontología, Ciudad Universitaria UCV, Los Chaguaramos, Caracas, Venezuela 1031. caromemo@gmail.com +584122379208 